\title{
Evaluation of the hydrodynamic pressure of cavitation impacts from stress-strain analysis and geometry of individual pits
}

\author{
Davide Carnelli ${ }^{\mathrm{a}, *}$, Ayat Karimi ${ }^{\mathrm{a}}$, Jean-Pierre Franc ${ }^{\mathrm{b}}$ \\ ${ }^{a}$ Institute of Condensed Matter Physics (ICMP), Swiss Federal Institute of Technology Lausanne (EPFL), Batiment PH, Station 3, CH-1015 Lausanne, Switzerland \\ ${ }^{\mathrm{b}}$ Laboratory of Geophysical and Industrial Flows (LEGI), Grenoble Institute of Technology, BP 53, 38041 Grenoble Cedex 9, France
}

\begin{abstract}
The present paper focuses on the development of an inverse procedure able to infer the hydrodynamic pressure distribution acting on the material surface and responsible for the creation of the cavitation pit based on the stress in a cavitation pit and its geometrical features. To achieve this goal, experimental pitting and nanoindentation measurement techniques were used together with a model of pit formation based on a Gaussian distribution of the hydrodynamic impact pressure pulse.

The pitting tests were performed at four different operating pressures on aluminum alloy samples and the geometrical characteristics of the pits were measured. Then the strain, stress, and load in a cavitation pit were quantified by coupling the pitting test analysis with the material information obtained via the indentation tests. Finally, a Gaussian distribution of the hydrodynamic impact pressure on the material surface has been hypothesized and the peak of this distribution as well as its width have been inferred.

This procedure, allowing the evaluation of the hydrodynamic impact pressure and load responsible for the material erosion and the deduction of insightful information on the flow aggressiveness at different operating conditions, could certainly represent a significant step in developing a technique able to evaluate the cavitation intensity from pitting tests.
\end{abstract}

\section{Introduction}

Cavitation exerts on solid surfaces nearby a particular solicitation characterized by repeated and randomly distributed stress pulses. Several works have focused on the estimation of the cavitation intensity or flow aggressiveness of a cavitating flow with the long term goal to model the material response to cavitation erosion solicitations and predict mass loss as a function of exposure time [1-5].

Undoubtedly, knowing the spectrum of impact loads could give precious information in terms of the flow aggressiveness and thus the material erosion. In principle, the simplest way to get this information would be to consider the use of conventional pressure sensors $[4,6,7]$. In the literature, different techniques have been developed for the direct measurement of cavitation collapse pulses, such as ceramics pressure transducers [4,8-10], pressure transducers made of piezoelectric films (PVDF) $[4,6,11]$, and crystals of magnesium oxide [4,12,13]. However, these

\footnotetext{
* Correspondence to: Laboratory of Nanostructured \& Complex Matter (LPMC), Institute of Condensed Matter Physics (ICMP), Swiss Federal Institute of Technology Lausanne (EPFL), PH D2 344 (Batiment PH) - Station 3, CH-1015 Lausanne Switzerland. Tel.: +412169344 38; fax:+41216934470.

E-mail address: davide.carnelli@epfl.ch (D. Carnelli).
}

sensors may not necessarily meet the required conditions for an accurate measurement of the impact loads, in particular in terms of rise time and resonant frequency; further, the results are position sensitive, so an accurate preliminary evaluation of the effect of the cavitating flow in different positions of the system has to be considered; then, they need to be properly calibrated; finally, their size is big compared to collapse size and they may be easily damaged by cavitation themselves.

In a recent paper [14], our group proposed to use the material itself as a sensor able to measure the impact loads due to bubble collapses by performing experimental pitting and nanoindentation tests. Indeed, although pitting tests have been proved to be an insightful tool in the field of cavitation erosion $[15,16]$ and the analysis of pitted surfaces has been significantly improved in recent years, as nowadays various techniques (optical profilometry [17], laser profilometry [18], scanning electron microscopy [19], etc.) are available for the analysis of the pits and the measurement of their geometric features, no validated procedure was yet available to infer, by an inverse technique, the characteristics of the stress pulses from the measured geometric characteristics of the pits. Since a quantitative approach toward this direction would necessarily require to properly model the material behavior, instrumented indentation measurement techniques [20-22] have been adopted due to their potential in providing 
useful information in the assessment of the intensity of cavitation impacts thanks to the similarities between substrate deformation under liquid impact and under indentation testing, where complex multiaxial stress fields occur.

Carnelli et al. [14] showed how the coupling between pitting test experiments and the material's mechanical behavior extracted from nanoindentation measurements together with the development of advanced analytical procedures to analyze the experimental data allows the evaluation of the hydrodynamic aggressiveness of a cavitating flow in terms of the spectra of pit stresses and impact loads responsible for material erosion. In this work, the main development of the procedure illustrated above consists of the introduction of a model of pit formation based on a Gaussian distribution of the hydrodynamic impact pressure pulse. This distribution represents the hydrodynamic pressure acting on the material surface and responsible for the creation of the cavitation pit. Further developments with respect to the previous paper from our group are (i) the use of a model which takes into consideration the effect of strain rate on the material's mechanical properties obtained by quasistatic indentation tests; (ii) the discussion of the role of the pitting rate at the different operating pressures in the hydrodynamic aggressiveness of a cavitating flow.

The scientific question addressed in the present work is whether an inverse procedure able to infer the hydrodynamic pressure distribution based on the stress in a cavitation pit and its geometrical features would be possible.

\section{Materials and methods}

The material selected for testing in this work was the aluminum alloy 7075-T651. For the sample preparation the procedure reported in [14] was followed.

\subsection{Pitting test experiments and analysis}

Pitting tests were conducted in a hydrodynamic tunnel described in detail in [23]. The test section is axisymmetric and is made of a nozzle of $16 \mathrm{~mm}$ in diameter which generates a high velocity flow followed by a radial divergent of $2.5 \mathrm{~mm}$ in thickness. Cavitation appears in the form of a cavity attached to the nozzle exit and damage to the samples facing the nozzle is concentrated in a circular ring centered on the cavity closure. The maximum operating pressure of the tunnel is $40 \mathrm{bar}$, which corresponds to a maximum velocity of $90 \mathrm{~m} / \mathrm{s}$. Tests were carried out at four different velocities between $45 \mathrm{~m} / \mathrm{s}$ (corresponding to an upstream pressure of $10 \mathrm{bar}$ ) and $90 \mathrm{~m} / \mathrm{s}$ at constant cavitation number: this ensures that the cavity length is almost constant and that a similarity exists between the different flows at different velocities. For each flow velocity, the test duration was adjusted in order to avoid any overlapping of pits which would result in measuring errors. The exposure time was only a few seconds for the tested alloy at the maximum velocity and was about $1 \mathrm{~min}$ at the lower velocity. For example, Fig. 1 shows a perspective view of the surface of a sample subjected to pitting test at 40 bar pressure. The reader is referred to Franc et al. [24] for further details on the experimental set-up and tests.

The analysis of a pitting test, consisting of the determination of the center coordinates, the maximum depth, the equivalent diameter, and the deformed volume of each pit by means of a conventional contact profilometer, is performed as in Carnelli et al. [14]. Here, we would only remark that, in order to identify the pits, a cut-off depth of $0.5 \mu \mathrm{m}$ below the original material surface is applied when post-processing the measured surface. This choice was considered to give a satisfactory account of the pitted surface. Therefore, pit size is defined as the equivalent

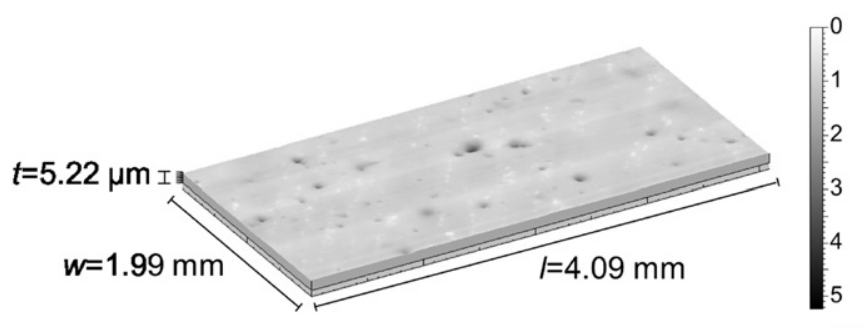

$\mu \mathrm{m}$

Fig. 1. Example of the surface of a sample subjected to pitting test at $40 \mathrm{bar}$ pressure. The pit depths are visualized in gray scale. A cut-off depth of $0.5 \mu \mathrm{m}$ below the original material surface was chosen. The sampled area width $w$ and length $l$ are reported too.

diameter of the section of the pit by the plane $x=0.5 \mu \mathrm{m}$, pit depth is counted from the original material surface $x=0$, and volume calculation is defined as in Franc [23]. For further details on the influence of choice of the threshold on the pitting analysis outcomes the reader is referred to Franc et al. [24].

\subsection{Nanoindentation experiments and analysis}

Nanoindentation experiments were performed using the Nano Indenter XP (Agilent Technologies Inc.) and following the same procedure reported in [14].

The aim of the indentation tests was to get direct knowledge of the mechanical behavior of the aluminum alloy subjected to testing in this work. This goal is reached by adopting the existing contact mechanics framework to deduce the relationship for stress and strain from indentation tests. Indeed, the definition of true indentation stress is [25]

$\sigma=\frac{1}{\psi} \frac{P}{A_{c}}$

where $P$ is the indentation load, $A_{c}$ is the projected area of contact, and $\psi$ is the constraint factor introduced by Francis [25], which has been found to be $\sim 1.11$ when a material is in the purely elastic regime, and increases in the elastic-plastic regime up to $\sim 2.87$ for full plasticity. Further, Tabor [26] suggested that the equivalent uniaxial strain at the indenter contact edge can be expressed by

$\varepsilon=0.2 \frac{a_{c}}{R}$

In the previous formulation, $a_{c}$ is the projected contact radius and $R$ is the radius of the spherical tip that is geometrically related to the contact depth $h_{c}$. For an extensive treatment of the procedure above, the reader is referred to Oliver and Pharr [20,27] for the nanoindentation data analysis and to Carnelli et al. [14] for the determination of stress-strain curves from load-displacement indentation tests.

With respect to the previous paper from our group [14], a different material model is adopted to identify the material parameters in this work. The Hollomon's law [28] is employed here to describe the nonlinear relationship between stress and strain and the work hardening phenomenon occurring in metals such as the aluminum alloy employed here. Hollomon's equation is a power law relationship between the stress $\sigma$ and the strain $\varepsilon$ : $\sigma=K \varepsilon^{n}$

where $K$ is the strength index and $n$ the strain hardening index, which describes the hardening behavior of the alloy. Eq. (3) can be conveniently rewritten as

$\sigma=\sigma_{0}\left(\frac{\varepsilon}{\varepsilon_{0}}\right)^{n}$ 
$\sigma_{0}$ being the stress related to the reference strain $\varepsilon_{0}$. The value $\varepsilon_{0}$ can be seen as a yield offset and was set at the commonly accepted value of strain of $0.2 \%$, which means that $\sigma_{0}$ represents the reference stress at $0.2 \%$ of plastic strain. In practice, $\sigma_{0}$ represents an estimate of the elastic limit of the material.

\subsection{Strain rate effect}

While nanoindentation tests were performed at a controlled strain rate of $0.05 \mathrm{~s}^{-1}$, cavitation erosion is known to be characterized by a higher strain rate, quantifiable in the order of $10^{3} \mathrm{~s}^{-1}$. In this work, the strain rate effect on the investigated material was taken into account by considering well developed theories presented in the literature.

The strain rate effect was introduced by applying the JohnsonCook constitutive model [29], which is an empirical model allowing the determination of the flow stress considering the effects of strain rate and temperature (not taken into account in this work):

$\sigma_{0}(\dot{\varepsilon})=\sigma_{0}\left(1+K \ln \dot{\varepsilon} / \dot{\varepsilon}_{0}\right)$

where $\sigma_{0}(\dot{\varepsilon})$ is the flow stress at high strain rate, $\sigma_{0}$ is the flow stress at the reference strain rate $\dot{\varepsilon}_{0}$ (here set to $0.05 \mathrm{~s}^{-1}$ ) and $K$ is a material constant which accounts for the sensitivity of the material mechanical behavior to strain rate. The model was adopted in order to predict the yield strength at a $10^{3} \mathrm{~s}^{-1}$ strain rate based on the yield stress at $0.05 \mathrm{~s}^{-1}$, obtained via the nanoindentation tests, and by best-fitting the experimental data from the literature on the same aluminum alloy employed here [30] to obtain the value of the parameter $K$.

\subsection{Evaluation of the stress and load in a cavitation pit}

As explained in [14], the pit stresses and loads of cavitation impacts can be conveniently evaluated following the analogy between material deformation under liquid impact and indentation testing. Assuming that a cavitation pit could be completely characterized by its depth and diameter and assuming a spherical cap geometry for modeling the cavitation pit (Fig. 2), Eq. (2), which gives the equivalent uniaxial strain at the indenter contact edge under spherical indentation [26], can be reformulated as

$\varepsilon_{P}=0.2 \frac{d_{P} h_{P}}{\left(d_{P} / 2\right)^{2}+h_{P}^{2}}$

where $d_{P}$ represents the diameter of a cavitation pit and $h_{P}$ its depth. Therefore, the measurement of pit depth and diameter

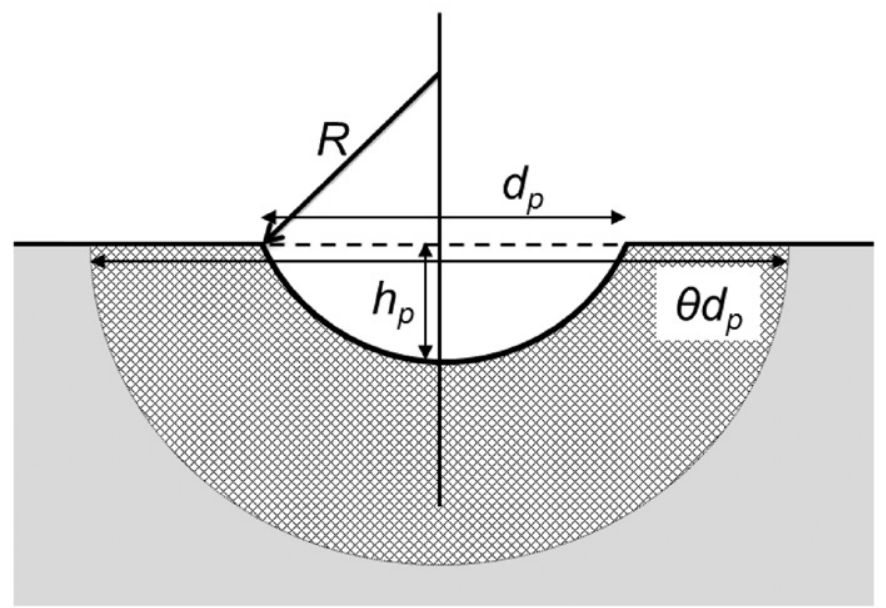

Fig. 2. Schematic of the spherical cap geometry adopted to model the cavitation pits. The extension of the plastically deformed area behind the pit is schematically reported. allows the determination of the strain at the border of a cavitation pit $\varepsilon_{P}$, corresponding to the strain at a distance $d_{P} / 2$ from the center of the pit in Fig. 2. By applying Eq. (6) in conjunction with the stress-strain relationship (Eq. (4)) corrected for the strain rate effect (Eq. (5)), the stress associated to a cavitation pit $\sigma_{P}$ was calculated. In addition, when the pit depth $h_{P}$ is small with respect to pit diameter $d_{P}$, Eq. (6) becomes

$\varepsilon_{P} \cong 0.8 \frac{h_{P}}{d_{P}}$

According to this model, the strain of a cavitation pit is measured by its aspect ratio $h_{P} / d_{P}$. Therefore the strain and the associated stress in a pit are proportional to its aspect ratio, and strain and stress will be almost the same for pits with different dimensions (i.e. different $h_{P}$ and $d_{P}$ ) but the same aspect ratio.

Once $\sigma_{P}$ is obtained, the impact load can be calculated by reformulating Eq. (1):

$L_{P}=\sigma_{P} A_{P} \psi$

In the previous equation, $A_{P}$ is the pit surface while the constraint factor $\psi$ has been set here to the value of 2.87 as full plasticity is expected to develop in the formation of a cavitation pit.

\subsection{Evaluation of the hydrodynamic pressure distribution acting on the material surface}

In order to find a direct correlation between the stress in a cavitation pit $\left(\sigma_{P}\right)$, the geometrical features of the cavitation pit $\left(h_{P}\right.$ and $d_{P}$ ), and the hydrodynamic pressure distribution acting on the material surface and responsible for the creation of the cavitation pit, a model of pit formation based on a Gaussian distribution of the hydrodynamic impact pressure pulse has been introduced (Fig. 3). This distribution can be expressed by the following equation:

$\frac{\sigma_{H}}{\sigma}=e^{\left(d / d_{H}\right)^{2}}$

where $\sigma_{H}$ is the peak of the hydrodynamic pressure distribution, $d_{H}$ is the hydrodynamic impact diameter, which represents the size of the impacted area on which the pressure pulse is applied, and $\sigma$ is the pressure at the radial distance $r=d / 2$ from the axis.

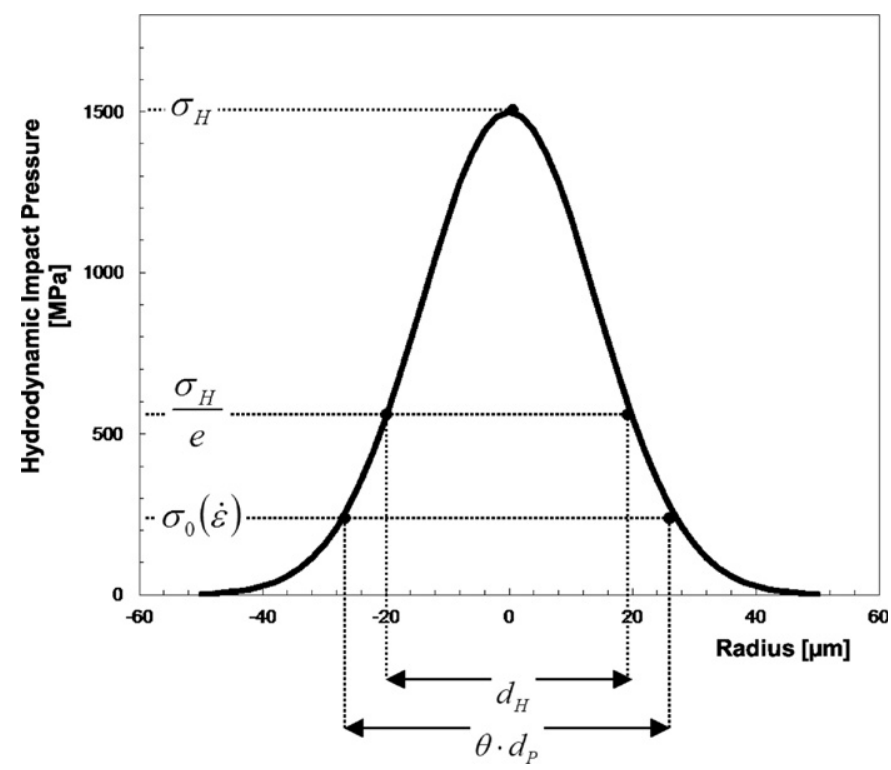

Fig. 3. Model of pit formation based on a Gaussian shape of the hydrodynamic impact pressure pulse acting on the material surface. 
By adopting the previous formulation, the impact load is equal to

$L_{H}=\int_{r=0}^{\infty} \sigma 2 \pi r d r=\sigma_{H} \pi \frac{d_{H}^{2}}{4}$

The values of the peak $\sigma_{H}$ of the hydrodynamic pressure distribution and hydrodynamic impact diameter $d_{H}$ have been inferred by considering that (i) the load $L_{P}$ calculated via the hydrodynamic pressure pulse (Eq. (10)) is equal to the load $L_{H}$ measured from the pit stress $\sigma_{P}$ (Eq. (8)), i.e. $L_{P}=L_{H}=L$; (ii) the value of the hydrodynamic pressure distribution is equal to the yield stress $\sigma_{0}(\dot{\varepsilon})$ of the material at the boundary of the plastic deformation on the material surface (Figs. 2 and 3): this occurs approximately at a distance of $\theta d_{P}$ from the center of impact, $\theta$ being a metallurgical parameter representing the shape factor of the strain profile inside the material [25]. This last parameter, which is related to the stain hardening index $n$, has been measured by nanoindentation tests as well and found to be equal to 1.52 . In other words, condition (i) means that the impact load is equal to that given by the nanoindentation approach, and condition (ii) means that the yield strength is reached at the limit between the plastically and elastically deformed regions. Conditions (i) and (ii) can be mathematically expressed by the following equations:

$\sigma_{H} \pi \frac{d_{H}^{2}}{4}=L_{P}$

$\frac{\sigma_{H}}{\sigma_{0}(\dot{\varepsilon})}=e^{\left(\vartheta d_{P} / d_{H}\right)^{2}}$

The system has been solved by using a least square regression method using the "Solver" tool of Excel. As the procedure detailed above was systematically applied to each pit, the distributions of the peaks of the hydrodynamic impact pressure $\sigma_{H}$ and hydrodynamic impact diameter $d_{H}$ were then obtained.

\section{Results}

In order to apply the procedure presented in Sections 2.4 and 2.5 to quantify the stress and load of cavitation impacts and the hydrodynamic pressure distribution, a proper mechanical modeling of the material behavior is required. The loading-unloading indentation curves on an aluminum alloy sample are reported in Fig. 4a. The pronounced hysteresis of the loading-unloading cycles indicates the presence of inelastic material behavior. The data show a high degree of reproducibility of the curves in both the loading and unloading branches in different locations on the sample surface. Fig. $4 \mathrm{~b}$ reports the average stress-strain curve obtained from the elaboration of the loading-unloading indentation curves (Section 2.2). The trend shows an initial elastic behavior followed by a region of plastic flow with material hardening. The Hollomon law (Eq. (4)) best-fitting the experimental stress-strain curves is depicted in Fig. 4b too. The adopted material model fits extremely well the extended work hardening region occurring in this alloy, while the initial elastic range of the material behavior is less well captured, as expected since the elastic deformation of the material is not explicitly taken into account by this model. The fitting results are presented in detail in Table 1, where the mean value and standard deviation of the fitting parameters $\sigma_{0}$ and $n$ over the 25 experimental curves are reported. The stress-strain curve corrected for the strain rate effect is also shown in Fig. 4b. The values of the constants adopted in the Johnson-Cook model to take into account the strain rate effect on the aluminum alloy mechanical properties are reported in Table 2, where the quasi-static yield stresses $\sigma_{0}$ and the
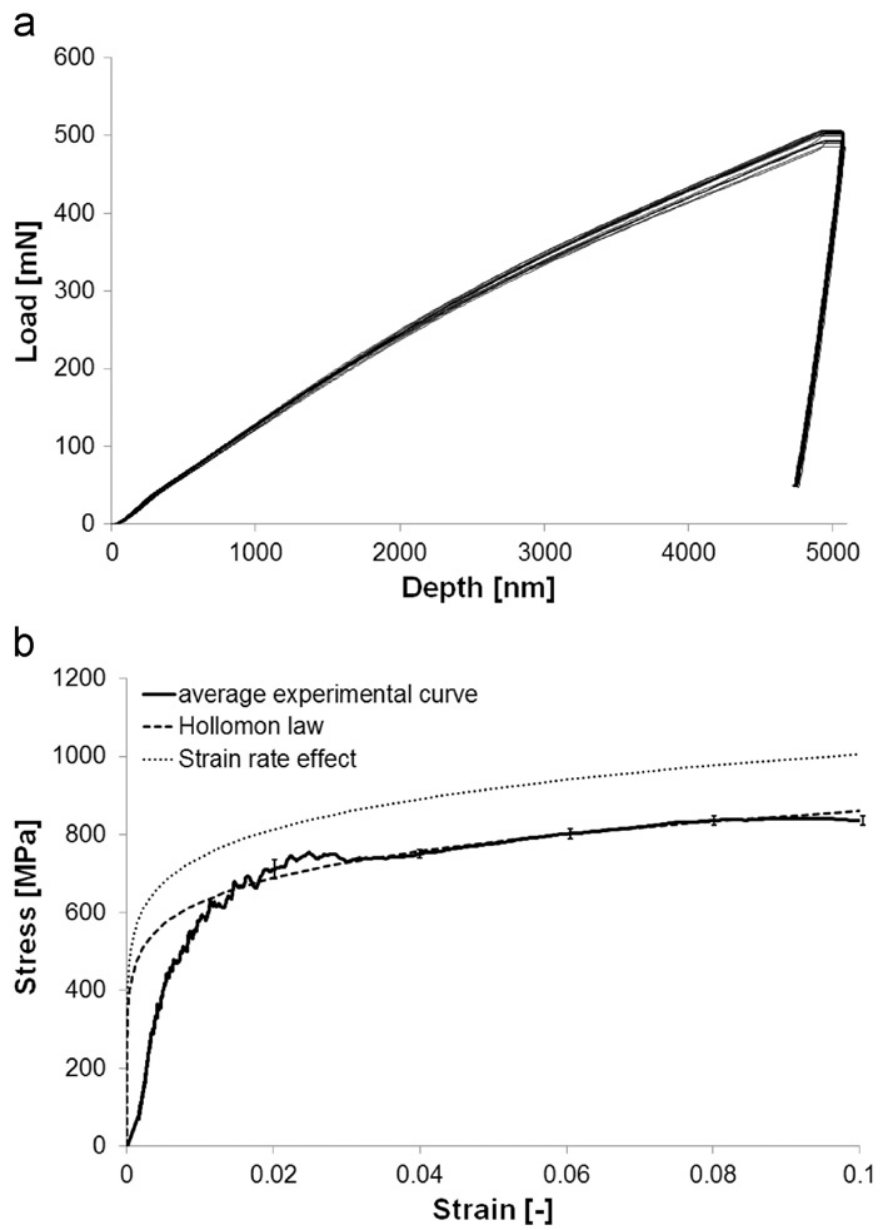

Fig. 4. (a) Load-penetration depth curves obtained from the $5 \times 5$ indentation tests at $5 \mu \mathrm{m}$ maximum depth on the aluminum alloy. (b) Average stress-strain curve obtained from the elaboration of the 25 loading-unloading indentation curves (continuum line). Standard deviations are calculated at 0.02 deformation intervals. The Hollomon law trend (Eq. (4)) identified from best-fitting of the experimental curves is reported (dashed line). The effect of strain rate on the material mechanical behavior is also shown (dotted line).

Table 1

Results of the best-fitting of the experimental stress-strain curve with the Hollomon law (Eq. (4)). The mean value and standard deviation of the fitting parameters $\sigma_{0}$ and $n$ over the 25 experimental curves are reported together with the coefficient of determination $R^{2}$.

\begin{tabular}{lll}
\hline$\sigma_{0}(\mathrm{MPa})$ & $n$ (dimensionless) & $R^{2}$ \\
\hline $514 \pm 12$ & $0.14 \pm 0.01$ & 0.97 \\
\hline
\end{tabular}

Table 2

Parameters adopted in the application of the Johnson-Cook constitutive model (Eq. (5)). The last column reports the yield stress obtained at $10^{3} \mathrm{~s}^{-1}$ strain rate $\left(\sigma_{0}(\dot{\varepsilon})\right)$.

\begin{tabular}{lllll}
\hline$\sigma_{0}(\mathrm{MPa})$ & $\dot{\varepsilon}_{0}\left(\mathrm{~s}^{-1}\right)$ & $\dot{\varepsilon}\left(\mathrm{s}^{-1}\right)$ & $K($ dimensionless $)$ & $\sigma_{0}(\dot{\varepsilon})(\mathrm{MPa})$ \\
\hline 514 & 0.05 & $10^{3}$ & 0.017 & 600 \\
\hline
\end{tabular}

corresponding value obtained at $10^{3} \mathrm{~s}^{-1}$ strain rate $\left(\sigma_{0}(\dot{\varepsilon})\right)$ can be directly compared.

As illustrated in Section 2.4, the correspondence between pit geometry and strain of a cavitation pit determined by Eq. (6) coupled with the stress-strain relationship in Eq. (4) corrected for the strain rate effect (Eq. (5)) allows obtaining the stress $\sigma_{P}$ in a 
cavitation pit for each impact. A plot of the pit stress $\sigma_{P}$-pit diameter $d_{P}$ correlation for the four tested upstream pressures is reported in Fig. 5. The associated strains are also shown. Each point represents one pit and a total number of 782 pits are plotted, corresponding to the four different values of the upstream pressures $(10,15,20$, and 40 bar).

The results of the inverse procedure performed to get the hydrodynamic pressure distribution acting on the material surface based on the pit stress $\sigma_{P}$ and the geometrical features of a cavitation pit are reported in Fig. 6a, where the peak $\sigma_{H}$ of the hydrodynamic pressure distribution is plotted as a function of the hydrodynamic impact diameter $d_{H}$. By comparing the trend in Fig. 6a with the one in Fig. 5, representing the pit stress-pit diameter correlation, immediately it can be noticed that the peak of the hydrodynamic pressure distribution is in many cases remarkably different from the corresponding pit stress: in more detail, an amplification of the pressure is particularly evident when small diameters are involved, as the maximum value of the distribution increases from $1065 \mathrm{MPa}$ in Fig. 5 to $1630 \mathrm{MPa}$ in Fig. 6a (about 53\%); instead, for big diameters, the difference in the pressure is much less, as the minimum value of the pressure distribution increases from $714 \mathrm{MPa}$ in Fig. 5 to just $886 \mathrm{MPa}$ in Fig. 6a (about 24\%). Basically the same considerations can be made regarding the hydrodynamic impact diameter, which remarkably increases for low values of $\sigma_{H}$ while it is virtually equal to the pit diameter $d_{P}$ for high values of $\sigma_{H}$.

A plot of impact load $L$ vs. hydrodynamic impact diameter $d_{H}$ is also shown (Fig. 6b). A strong correlation with the power law is found between the impact load $L$ and the hydrodynamic impact diameter $d_{H}$. The impact load correlation with diameter does not depend significantly upon the operating conditions in the present range of investigation. Indeed, by performing a linear regression analysis on the four sets of data, the value of the exponent of the power law trend for $10,15,20$, and 40 bar upstream pressures were $1.87,1.88,1.88$, and 1.90 , respectively. Therefore, the exponent is slightly lower than two and the impact load was found to have a strong, almost quadratic correlation with hydrodynamic impact diameter. A coefficient of determination $R^{2}$ higher than 0.99 was found.

Based on the data in Fig. 6a and b, a histogram of the number of impacts vs. peak of hydrodynamic pressure distribution $\sigma_{H}$ was drawn by dividing the whole spectrum of pressure peaks in 25 levels from the lowest $\left(\sigma_{H}^{\min }=886 \mathrm{MPa}\right)$ to the highest $\left(\sigma_{H}^{\max }=1630\right.$ $\mathrm{MPa}$ ) pressure recorded and considering the number of impacts

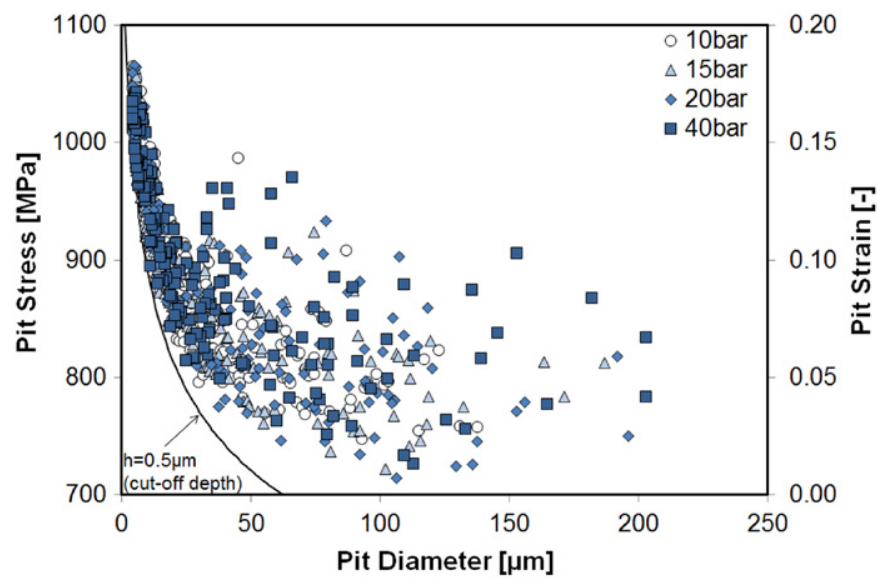

Fig. 5. Results of the analytical procedure adopted to determine the stress and load in a cavitation pit: plot of the pit stress $\sigma_{P}$-pit diameter $d_{P}$ correlation for the four upstream pressures. The corresponding pit strains $\varepsilon_{P}$ are reported in the secondary vertical axis. The stress and strains limit curve corresponding to a pit depth equal to $0.5 \mu \mathrm{m}$ cut-off depth is also reported.
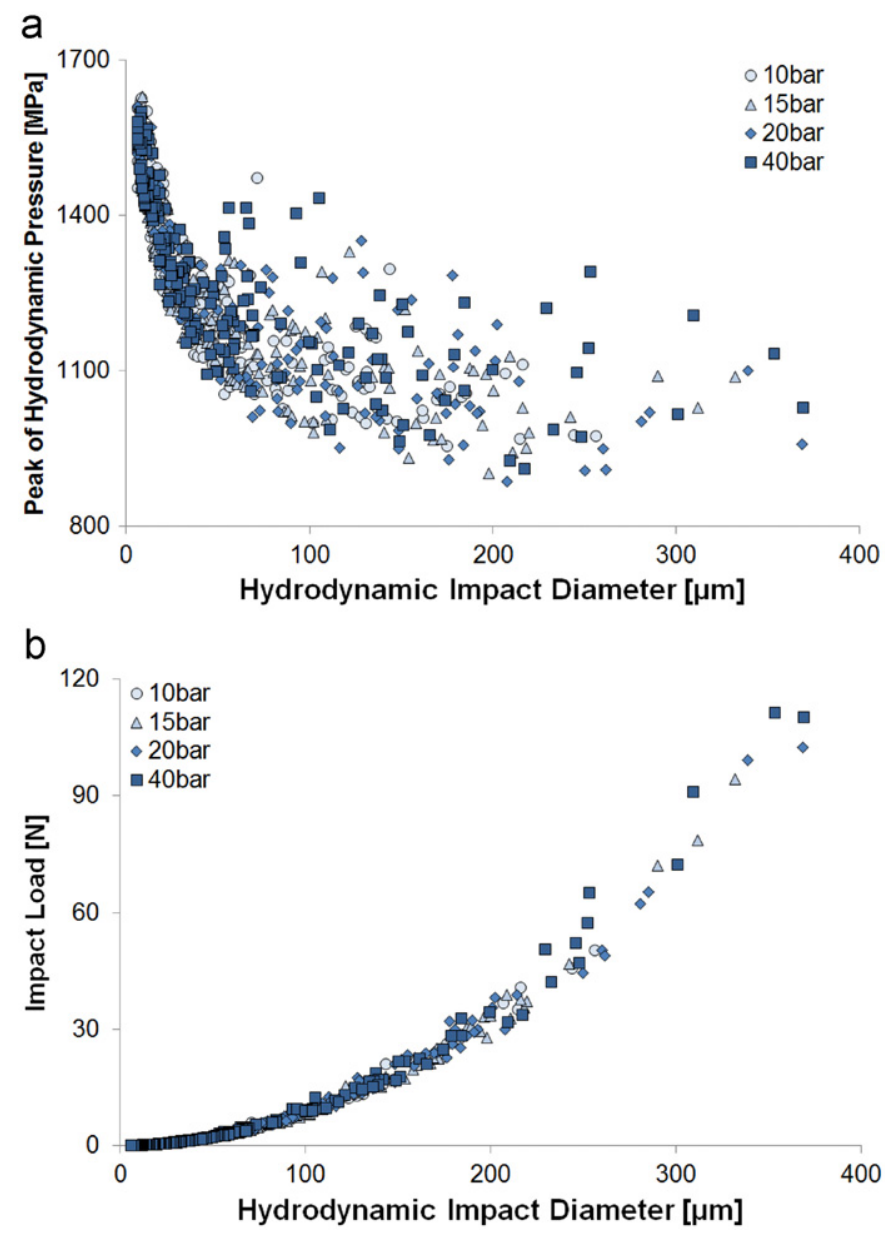

Fig. 6. Results of the inverse procedure adopted to determine the hydrodynamic pressure distribution acting on the material surface. (a) Representation of the peak of the hydrodynamic pressure distribution $\sigma_{H}$ as a function of the hydrodynamic impact diameter $d_{H}$. (b) Representation of the impact load $L$-hydrodynamic impact diameter $d_{H}$ trends at the four upstream pressures.

$N_{i}$ belonging to each interval with respect to their total number $N_{t}$ (Fig. 7a). Therefore, this plot represents the probability density function of the hydrodynamic pressure pulses amplitudes. The same procedure was employed to calculate the histogram of the number of impacts vs. impact load $L$ (Fig. 7b). In this case, the whole spectrum of impact loads was divided into 25 levels from the lowest $\left(L^{\min }=0.05 \mathrm{~N}\right)$ to the highest $\left(L^{\max }=111 \mathrm{~N}\right)$ load recorded.

The obtained maximum loads at the four investigated upstream pressures are reported in Table 3. As the maximum loads $L^{\max }$ range between $\sim 50 \mathrm{~N}$ at 10 bar upstream pressure and $\sim 111 \mathrm{~N}$ at 40 bar upstream pressure, a correlation with the upstream pressures is evidenced. A further indication in this sense can be found if we extend this analysis to a broader number of impacts: as an example, Table 3 reports the average load value of the $10 \%$ stronger impacts obtained at the four different upstream pressures. In this case a higher differentiation between the 15,20 and 40 bar results is found.

\section{Discussion and conclusion}

This paper deals with the evaluation of the hydrodynamic pressure distribution acting on the material surface and responsible for the creation of the cavitation pit based on the measurements of the stress and load in a pit. To pursuit this goal, pitting and nanoindentation mechanical tests on aluminum alloy samples were 
a

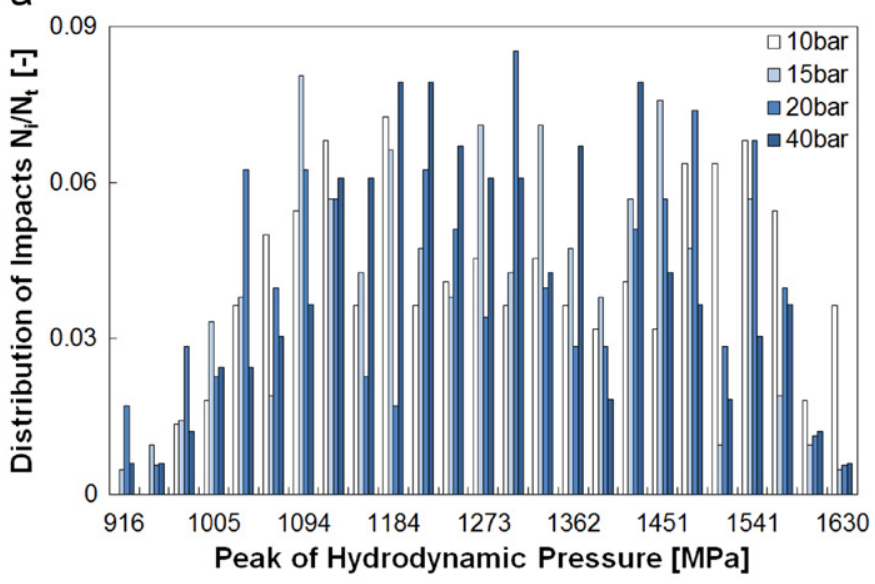

b

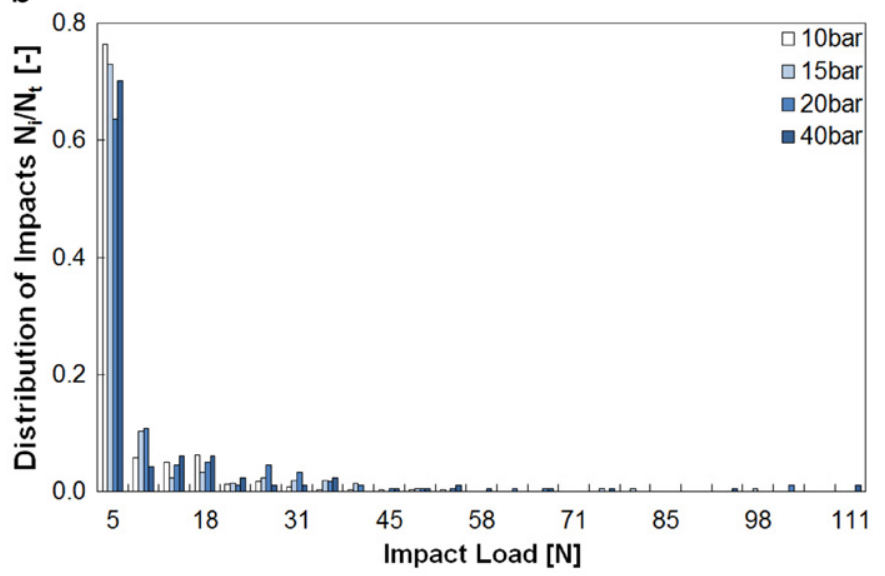

Fig. 7. Statistical distribution of the peaks of hydrodynamic pressure distribution and impact loads. (a) Histogram of the number of impacts vs. peak of hydrodynamic pressure distribution $\sigma_{H}$. (b) Histogram of the number of impacts vs. impact load $L$ for the four tested upstream pressures. $N_{i}$ represents the number of impacts belonging to a certain interval; whereas, $N_{t}$ is the total number of impacts detected at the four different upstream pressures.

Table 3

Maximum loads $\left(L^{\max }\right)$ and average load value of the $10 \%$ stronger impacts $\left(L^{10 \%}\right)$ for the four tested upstream pressures.

\begin{tabular}{llccc}
\hline & 10 bar & 15 bar & 20 bar & 40 bar \\
\hline$L^{\max }(\mathrm{N})$ & 50.4 & 94.2 & 102 & 111 \\
$L^{10 \%}(\mathrm{~N})$ & 22.7 & 38.9 & 45.9 & 59.4 \\
\hline
\end{tabular}

conducted and a model of pit formation based on a Gaussian distribution of the hydrodynamic impact pressure pulse has been introduced.

The benefit of introducing this model of impact load includes the ability to distinguish between pit diameter (i.e. the diameter of the plastic deformation) and impact load diameter (i.e. the size of the impacted area on which the computed impact load is applied, typically that of the re-entrant jet) and between pit stress (the stress evaluated in the alloy at the indenter contact edge, as reported in Section 2.4) and hydrodynamic impact pressure (the pressure acting on the material surface and responsible for the creation of the cavitation pit). Indeed, pit diameter $d_{P}$ and pit stress $\sigma_{P}$ are material dependent quantities as the same hydrodynamic impact is expected to generate a different pit on two different materials. Roughly speaking, a smaller and shallower pit will be formed in a harder material. For a deterministic prediction of cavitation erosion, it is essential to find out the hydrodynamic pressure distribution generated on the wall by each collapsing bubble. When using a Gaussian law to model this pressure distribution (as supposed in this paper), the hydrodynamic impact is characterized by two parameters, namely the peak of hydrodynamic pressure $\sigma_{H}$ and the diameter of the hydrodynamic impact $d_{H}$. In contrast to $\sigma_{P}$ and $d_{P}$ which depend upon the material, the two quantities $\sigma_{H}$ and $d_{H}$ are supposed to be purely hydrodynamic quantities. Since they are determined on the basis of pitting tests conducted on a given material, it is important to develop a procedure to derive the two hydrodynamic quantities $\sigma_{H}$ and $d_{H}$ from the two material dependent quantities $\sigma_{P}$ and $d_{P}$. This was the major motivation of the present paper. Further work is needed to confirm that the so-defined $\sigma_{H}$ and $d_{H}$ values are actually material independent.

Depth sensing nanoindentation measurements were employed to extract the mechanical properties of the material exposed to cavitation erosion. The loading-unloading indentation curves (Fig. 4a) show a high degree of reproducibility. Further evidence supporting this statement regards the obtained value of the maximum loads $P_{\max }$ over the whole set of indentation curves, which is equal to $499.5 \pm 6.9 \mathrm{mN}$. The deduced COV (coefficient of variation, defined as the ratio between standard deviation and mean value) is 0.014 . With respect to this, we can also underline how the obtained value of the maximum indentation load $P_{\max }$ obtained for the $5 \mu \mathrm{m}$ deep indentations represents an intermediate value between the lowest $\left(L_{\min }=0.05 \mathrm{~N}\right)$ and the highest $\left(L_{\max }=111 \mathrm{~N}\right)$ loads recorded. Therefore, it can be speculated that the same accuracy in the results has been obtained for the shallow pits as well as for the bigger ones. Anyway, this does not represent an important issue since the extraction of the impact stress from the pit geometrical parameters is mediated by the stress-strain curve and not performed directly by the indentation tests, as it would be if a test is carried out in order to "replicate" a pit, in terms of its depth and diameter, and directly measure the load required to create it.

The obtained pit stress-pit diameter distribution (Fig. 5), showing an increase in the stress as the pit size decreases, has been found by other researchers too [11]. In this paper, the shape of this distribution is directly linked to the methodology adopted to calculate the strains from the pitting geometry: as shown in Eq. (7), the impact strain $\varepsilon_{P}$ in a pit is proportional to its aspect ratio $h_{P} / d_{P}$, so it is directly proportional to pit depth $h_{P}$ but inversely proportional to pit diameter $d_{P}$. As the increase in the pit diameter was higher than the increase in the pit depth when comparing shallow and deep pits, the strains evaluated for large diameters are likely to be lower than the ones corresponding to small diameters. Thus, the stresses based on larger residual indents areas (high intensity impacts) will be lower than those based on the very small pits. Further, the effect of the measuring technique, and in particular the depth threshold of $0.5 \mu \mathrm{m}$, is evidenced in Fig. 5 by the continuum line, which represents the theoretical stress and strains curve corresponding to a pit depth of $0.5 \mu \mathrm{m}$, i.e. equal to the cut-off depth. Actually, this is a limit curve since stresses and strains below this curve cannot be detected. Thus the correlation between pit stress and pit diameter in the left-hand part of the plot could in part be an artifact connected to the measuring technique and the associated cut-off depth rather than a characteristic of cavitation erosion pits. Further investigations in this sense are required. The same considerations hold for the hydrodynamic pressure distribution trend with hydrodynamic impact diameter $d_{H}$ (Fig. 6a).

This situation does not hold if, instead of the pit stress $\sigma_{P}$, we consider the impact load $L$ trend with hydrodynamic impact diameter, reported in Fig. 6b: the impact load clearly increases with the diameter in an almost quadratic way. Further, the impact load-pit volume correlation shows a similar trend, with the 
impact load increasing almost linearly with pit volume, as shown in [14] (data not reported). Therefore, we can conclude that whereas pit stresses are proportional to the pits aspect ratio, impact loads are proportional to pit volume or to pit diameter squared.

The maximum values of the impact loads reported in Table 3 are in reasonable agreement with data from the literature concerning the direct measurement of cavitation erosion pulses. As an example, Momma and Lichtarowicz [11] developed a piezoelectric pressure transducer using PVDF film. Pulse height distributions were measured and counted in three different operating conditions obtaining maximum values between 60 and $70 \mathrm{~N}$. Using PVDF transducers as well, Soyama et al. [6] measured maximum impact loads of $\sim 200 \mathrm{~N}$ in a cavitating jet apparatus for a jet velocity at the nozzle outlet in the range of $126-155 \mathrm{~m} / \mathrm{s}$. By using a pressure-sensitive piezoelectric ceramic disk, Okada et al. [8] found a maximum impact load of $22.1 \mathrm{~N}$ for a venturi test with a duration of $300 \mathrm{~s}$ and $25 \mathrm{~m} / \mathrm{s}$ velocity. More recently, Hattori et al. [7] measured the bubble collapse impact loads by using a piezoceramic transducer as well. A distribution of the impact loads was obtained by detecting several thousand counts both for small and large impact loads at flow velocities of up to $184 \mathrm{~m} / \mathrm{s}$. A maximum impact load of $21 \mathrm{~N}$ was detected. On the other hand, Franc et al. [31] estimated the impact loads by means of a commercial piezoelectric pressure sensor and higher maximum values, in the range of 100-500 N, were estimated for the same experimental device as the one used here. Therefore, as already underlined in [31] there are significant discrepancies between estimates of impact loads obtained by different techniques even for the same cavitating flow. A possible development could be to introduce energy considerations in the evaluation of the impact loads both with pressure transducer and with the analytical procedure reported in this paper in order to reconcile the impact load data. Work in this direction is currently in progress.

As already reported in Carnelli et al. [14], no clear dependence on the flow conditions is found neither in terms of pits geometrical features only (pit depth, diameter, volume and their correlation) nor when explicitly introducing the materials' mechanical behavior to evaluate the pit stress and load. In the pit stress-pit diameter distribution (Fig. 5) and peak of the hydrodynamic pressure-hydrodynamic diameter distribution (Fig. 6a), as well as in the histogram of the number of impacts vs. peak of hydrodynamic pressure (Fig. 7a), no clear distinction between the data at the four different operating pressure stands out. Whereas, the impact load histograms at the four different operating pressures (Fig. 7b) together with the results reported in Table 3 can give some insights about the operating conditions. The difference in the flow aggressiveness can be assessed by considering maximum loads $L^{\max }$ and average load value of the $10 \%$ stronger impacts $L^{10 \%}$ for the four tested upstream pressures obtained in the present work. These results can be interpreted in statistical terms. Indeed, while weak impacts occur at all the upstream pressure levels and therefore the loads related to these impacts are basically superimposed in the different operating conditions, the occurrence of intense events, which corresponds to large values of the pit volume and high impact loads associated with them, is more likely to occur when the upstream pressure (i.e. the flow velocity) is high. Recalling that the impact loadhydrodynamic impact diameter trends of Fig. $6 \mathrm{~b}$ do not depend significantly upon the operating conditions as the exponent values are very close to each other (see Section 3), we can infer that the information on the fluid flow obtained at 40 bar pressure consists basically of the information at the lower pressures plus the occurrence of intense events which are not likely to occur unless the global energy of the system is high enough. Therefore, the maximum values of the recorded impact load for each pressure condition are correlated to the pressure of the cavitating tunnel and they can be hypothesized to be reliable indicators of the flow aggressiveness in different operating conditions, while the global trend of impacts loads is basically independent of the fluid conditions. Further, impact loads seem a better indicator than stresses in the material or hydrodynamic pressure in order to evaluate the flow aggressiveness in different operating conditions.

Nevertheless, a difference in the four operating pressures clearly stands out if we introduce the pitting rates, defined as the number of detected pits $N$ for unit exposure time $t$ and unit surface $A_{0}$ :

$P R=\frac{N^{i}}{t^{i} A_{0}^{i}}$

In the previous formulation, the index $i$ represents the considered hydrodynamic condition. The pitting rate at the four operating pressures is plotted as a function of the flow velocity in the tunnel in Fig. 8. A strong power law trend is evidenced in the plot. As clearly seen, the pitting rate depends on the flow conditions: indeed, for the Al sample, increasing by a factor two the flow velocity in the tunnel (from $45 \mathrm{~m} / \mathrm{s}$ to $90 \mathrm{~m} / \mathrm{s}$ ) corresponds to a pitting rate $\sim 50$ times higher. The effect of pitting rate is also clearly evident when the histograms of the peak of hydrodynamic pressure distribution $\sigma_{H}$ (Fig. 7a) are multiplied by the pitting rate, as reported in Fig. 9. In this case, about two orders of magnitude of difference between the two extreme upstream pressures have been found in terms of peak of hydrodynamic pressure $\sigma_{H}$. Therefore, the analysis of the effect of pitting rate on the obtained results allows stating that the increase in cavitation aggressiveness between $10 \mathrm{bar}$ and 40 bar seems to be related more to a strong increase in frequency than to an increase in the absolute values of pit strain, pit stress or peak of hydrodynamic pressure distribution. This remark is consistent with the findings reported by Franc et al. [24,31].

To conclude, in this work the coupling between pitting test analysis and material mechanical behavior extracted from nanoindentation measurements together with a proper model of the distribution of the hydrodynamic impact pressure pulse allowed us to measure the stress and load in a cavitation pit and to evaluate the peak of the hydrodynamic pressure distribution by means of an inverse procedure. In the authors' opinion, this development of the technique based on pitting and indentation

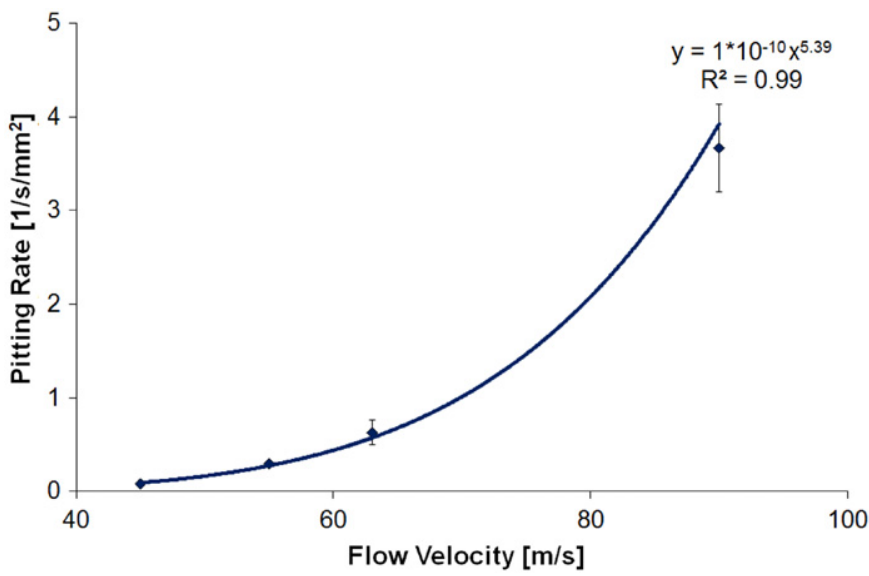

Fig. 8. Pitting rate as a function of the flow velocity in the tunnel for the $\mathrm{Al}$ sample. 10, 15, 20 and 40 bar operating pressures correspond to $45,55,63$ and $90 \mathrm{~m} / \mathrm{s}$ maximum velocity in the tunnel, respectively. Best-fitting with a power law trend is shown. Error bars are \pm one standard deviation calculated between the three/four $2 \times 4 \mathrm{~mm}$ surfaces analyzed in each Al sample. 


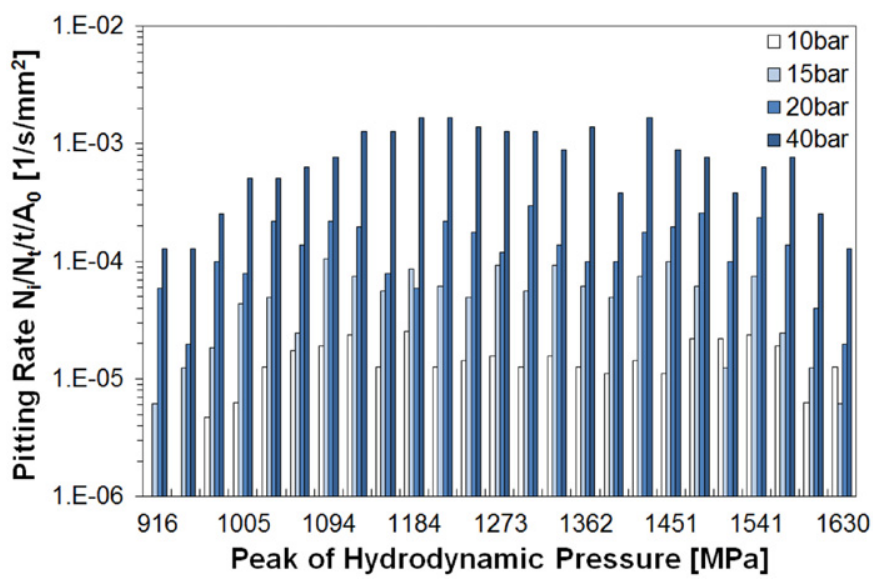

Fig. 9. Statistical distribution of the peaks of hydrodynamic pressure: histogram of the normalized number of impacts vs. peak of hydrodynamic pressure distribution $\sigma_{H}$ at the four tested upstream pressures. $N_{i}$ and $N_{t}$ are as in Fig. 7, $t$ is the exposure time, and $A_{0}$ the area of the analyzed surface. Note the semilogarithmic scale.

tests represents another important step in the investigation of cavitation erosion damage.

\section{Acknowledgments}

The authors wish to thank Dr. Ki-Han Kim from the Office of Naval Research (ONR) and Dr. Richard Vogelsong from the Office of Naval Research Global (ONRG) who supported this work. They are also very grateful to Dr. Georges L. Chahine (Dynaflow, Inc.), Dr. Farrel Martin (Naval Research Laboratory) and Dr. Martin Donnelly (NSWCCD) for fruitful discussions and to Michel Riondet for his contribution in the pitting tests.

\section{References}

[1] F.G. Hammitt, Cavitation erosion: the state of the art and predicting capability, Applied Mechanics Review 32 (1979) 665-675.

[2] A. Karimi, J.L. Martin, Cavitation erosion of materials, International Materials Reviews 31 (1986) 1-26.

[3] A. Karimi, W.R. Leo, Phenomenological model for cavitation erosion rate computation, Materials Science and Engineering 95 (1987) 1-14.

[4] J.-P. Franc, J. Michel, Cavitation erosion research in France: the state of the art Journal of Marine Science and Technology 2 (1997) 233-244.

[5] H. Kato, A. Konno, M. Maeda, H. Yamaguchi, Possibility of quantitative prediction of cavitation erosion without model test, Journal of Fluids Engineering 118 (1996) 582-588.
[6] H. Soyama, A. Lichtarowicz, T. Momma, E.J. Williams, A new calibration method for dynamically loaded transducers and its application to cavitation impact measurement, Journal of Fluids Engineering 120 (1998) 712-718.

[7] S. Hattori, M. Takinami, O. Tomoaki, Comparison of cavitation erosion rate with liquid impingement erosion rate, in: Proceedings of the 7th International Symposium on Cavitation CAV2009, Ann Arbor, Michigan, USA, 2009.

[8] T. Okada, Y. Iwai, S. Hattori, N. Tanimura, Relation between impact load and the damage produced by cavitation bubble collapse, Wear 184 (1995) 231-239.

[9] I.R. Jones, D.H. Edwards, An experimental study of the forces generated by the collapse of transient cavities in water, Journal of Fluid Mechanics 7 (1960) 596-609.

[10] S. Hattori, H. Mori, T. Okada, Quantitative evaluation of cavitation erosion, Journal of Fluids Engineering 120 (1998) 179-185.

[11] T. Momma, A. Lichtarowicz, A study of pressures and erosion produced by collapsing cavitation, Wear 186-187 (1995) 425-436.

[12] S. Hattori, K. Miyoshi, D.H. Bukley, T. Okada, Plastic deformation of a magnesium oxide $\{001\}$ surface produced by cavitation, in: Proceedings of the ASME/ASLE Tribology Conference, Pittsburgh, Pennsylvania, USA, 1986.

[13] T. Okada, S. Hattori, M. Shimizu, A fundamental study of cavitation erosion using a magnesium oxide single crystal (intensity and distribution of bubble collapse impact loads), Wear 186 (1995) 437-443.

[14] D. Carnelli, A. Karimi, J.-P. Franc, Application of spherical nanoindentation to determine the pressure of cavitation impacts from pitting tests, Journal of Materials Research 27 (2012) 91-99.

[15] R.T. Knapp, Recent investigations of the mechanics of cavitation and cavitation damage, Transactions of the ASME (1954) 1045-1054.

[16] R.T. Knapp, Accelerated field tests of cavitation intensity, Transactions of the ASME (1958) 91-102.

[17] B. Belahadji, J.-P. Franc, J.-M. Michel, A statistical analysis of cavitation erosion pits, Journal of Fluids Engineering 113 (1991) 700-706.

[18] R. Fortes Patella, J.L. Reboud, A. Archer, Cavitation damage measurement by 3D laser profilometry, Wear 246 (2000) 59-67.

[19] S.M. Ahmed, K. Hokkirigawa, Y. Ito, R. Oba, Scanning electron microscopy observation on the incubation period of vibratory cavitation erosion, Wear 142 (1991) 303-314.

[20] W.C. Oliver, G.M. Pharr, Measurement of hardness and elastic modulus by instrumented indentation: advances in understanding and refinements to methodology, Journal of Materials Research 19 (2004) 3-20.

[21] A.C. Fischer-Cripps, Nanoindentation, Springer Verlag, 2004.

[22] M.R. VanLandingham, Review of instrumented indentation, Journal of Research-National Institute of Standards and Technology 108 (2003) 249-266.

[23] J.-P. Franc, Incubation time and cavitation erosion rate of work-hardening materials, Journal of Fluids Engineering 131 (2009) 021303.

[24] J.P. Franc, M. Riondet, A. Karimi, G.L. Chahine, Material and velocity effects on cavitation erosion pitting, Wear 274-275 (2012) 248-259.

[25] H.A. Francis, Phenomenological analysis of plastic spherical indentation, Journal of Engineering Materials and Technology 98 (1976) 272-281.

[26] D. Tabor, The Hardness of Metals, Clarendon, Oxford, 1951.

[27] W.C. Oliver, G.M. Pharr, Improved technique for determining hardness and elastic modulus using load and displacement sensing indentation experiments, Journal of Materials Research 7 (1992) 1564-1583.

[28] J.H. Hollomon, Tensile deformation, AIME Transactions 12 (1945) 1-22.

[29] G.R. Johnson, W.H. Cook, A constitutive model and data for metals subjected to large strains, high strain rates and high temperatures, in: Proceedings of the 7th International Symposium on Ballistics, 1983, pp. 541-547.

[30] J.R. Pothnis, Y. Perla, H. Arya, N.K. Naik, High Strain Rate Tensile, Behavior of aluminum alloy 7075 T651 and IS 2062 mild steel, Journal of Engineering Materials and Technology 133 (2011) 021026.

[31] J.P. Franc, M. Riondet, A. Karimi, G.L. Chahine, Impact load measurements in an erosive cavitating flow, Journal of Fluids Engineering 133 (2011) 121301. 